Escuela de Derecho de la Universidad de Valparaíso. Chile.

Escuela de Derecho de la Universidad Diego Portales, Santiago, Chile. aDoctora en Derecho por la Universidad Autónoma de Madrid.

La autora declaró no tener conflictos de intereses en este manuscrito.

Recibido el 26 de julio de 2012, aceptado el 10 de enero de 2013

Correspondencia a: Sra. Alejandra Zúñiga Fajuri Errázuriz 2120. Valparaíso. Tel: 32-250 7014 / 32-250 7048 E-mail: alejandra.zuniga@ uv.cl

\section{Isapres, tribunal constitucional y distribución del derecho a cuidado sanitario}

\author{
ALEJANDRA ZÚÑIGA F. ${ }^{a}$
}

This paper analyzes the constitutional problems that the private health system has faced as a result of the recent decisions of the Constitutional Court and the Supreme Court of Chile in defense of the right to health care and nondiscrimination. It also reviews the comparative literature on health systems that have been successful in the task of reconciling the demands of equity and efficiency in the delivery of health care in the private health sector, in accordance with the constitutional principles of equality and nondiscrimination.

(Rev Med Chile 2013; 141: 514-518).

Key words: Constitution and By-laws; Delivery of Health Care; Patient rights; Social justice.

E 1 sistema privado de salud en Chile está en crisis. Las Instituciones de Salud Previsional (Isapres) no han sido capaces de responder a las exigencias de justicia generadas por la reforma del Acceso Universal de Garantías Explícitas (AUGE) y la última jurisprudencia de los tribunales, lo que ha significado una permanente avalancha de Recursos de Protección y Recursos de Inaplicabilidad que no parece sino aumentar cada día (casi 90\% del total del trabajo de las Cortes de Apelaciones de Santiago, Temuco y Valparaíso son sólo recursos contra las Isapres).

En este artículo se estudian los dos modelos de distribución de recursos sanitarios que están en la actualidad en pugna, así como las sentencias que los máximos tribunales han dictado en defensa de aquel modelo que comprende a la protección de la salud como un derecho que debe ser garantizado sin discriminación. Se revisa la literatura especializada en materia de sistemas sanitarios comparados exitosos en la tarea de compatibilizar las demandas de equidad y eficiencia en la prestación del recurso "cuidado sanitario".

\section{El modelo de distribución de recursos sanitarios del sistema privado de salud}

Al analizar los distintos criterios de selección de pacientes o de grupos sociales potencialmente beneficiarios de asistencia sanitaria, es necesario preguntarse qué diferencias son moralmente relevantes para el acceso diferencial a la asistencia sanitaria. Los siguientes principios han sido cada uno propuestos como principios materiales de justicia distributiva:

1. A cada persona una parte igual.

2. A cada persona de acuerdo con la necesidad.

3. A cada persona de acuerdo con el esfuerzo.

4. A cada persona de acuerdo con la contribución.

5. A cada persona de acuerdo con el mérito.

6. A cada persona de acuerdo con los intercambios del libre mercado (o de acuerdo a su capacidad de pago).

La mayoría de las sociedades invocan varios de estos principios materiales en el diseño de políticas públicas, apelando a diversos principios en 
diferentes contextos y esferas ${ }^{1}$. Pero, puesto que la distribución de recursos asociados al derecho a la protección de la salud es una preocupación primordial de las sociedades modernas en la medida en que influyen de manera radical en la preservación de nuestro estatus de ciudadanos en completo funcionamiento, se han visto como objetos apropiados de seguridad social y privada ${ }^{2}$.

Suele sostenerse que en Chile se mantienen vigentes dos modelos de adjudicación del recurso "cuidado sanitario". Uno que considera la provisión de acceso universal a la salud a través del sistema público ${ }^{3}$ y otro que considera que el criterio de distribución adecuado debe ser sólo la capacidad de pago, como ocurre con los seguros privados. Con todo, los organismos internacionales especializados, como la Organización Mundial de la Salud (OMS), consideran que el cuidado sanitario necesario para mantener una justa igualdad de oportunidades no debiera distribuirse conforme a la capacidad de pago, sino que en base al criterio de la "necesidad sanitaria"4.

En esta línea es posible evaluar críticamente este segundo modelo de distribución de recursos y su capacidad para responder a las demandas de igualdad emanadas tanto por los usuarios como por la reciente jurisprudencia del Tribunal Constitucional (TC) y la Corte Suprema (CS), desde la óptica de lo que en el sistema comparado se entiende como una correcta relación público-privada en materia de derecho a la protección de la salud. ¿Es legítimo permitir que las instituciones sanitarias, sean estas públicas o privadas, consideren criterios como la edad o el sexo del paciente para decidir sobre la adjudicación de bienes y servicios asociados al cuidado sanitario?

Sabemos que nuestro país mantiene vigente un sistema sanitario de tipo pluralista o mixto de consecuencias financieras fuertemente regresivas ${ }^{5}$. La naturaleza del sistema de salud privado -que es, básicamente, un sistema de seguro- se basa en la segregación entre los afiliados en relación con su riesgo asociado, disponiendo planes de salud con primas muy diferentes para mujeres y hombres, ancianos y jóvenes. Este sistema regresivo se ha mantenido debido a la imposibilidad política de modificarlo, tanto al momento de aprobarse la reforma sanitaria del año 2005, como posteriormente, lo que ha originado la actual avalancha de recursos de inaplicabilidad e inconstitucionalidad presentados ante el TC y Recursos de Protección interpuestos ante las Cortes de Apelaciones.

Desde las sentencias del TC $\mathrm{N}^{\circ} 1218-08$ y $\mathrm{N}^{\circ}$ 976-08, que declaran inaplicable la norma de la ley 18.933 de Isapres art. 38 , se ha generado una vasta jurisprudencia destinada a impedir que las aseguradoras privadas continúen, por la vía del empleo de tablas de factores de riegos, discriminando a las personas en razón de su sexo o edad. Cualquier tipo de alza en función de estos factores es inconstitucional en la medida que afecta los derechos a la igualdad, a la protección de la salud y a la seguridad social, reconocidos en la Constitución ${ }^{6}$. Por ello, la doctrina más reciente reconoce que las prestaciones que envuelven derechos sociales deben ser igualitarias y suficientes y deben estar, en último término, garantizadas por el Estado ${ }^{7}$.

En consecuencia, las Isapres tienen vedado afiliar o modificar el plan de salud de las personas en función de las tablas de factores derogadas. En los contratos vigentes, el efecto de la sentencia del 9 de agosto de 2010 debiera implicar que, al ser de tracto sucesivo y estar regulados por normas de orden público (que rigen in actum), el precio de los planes debiera valorarse sumando el precio del Plan AUGE más el costo base del plan complementario, sin posibilidad de aumentarlo en razón del factor sexo o edad.

\section{La evaluación de la justicia al modelo de distribución de las Isapres}

La nueva jurisprudencia considera que el modelo de distribución de recursos sanitarios vigente en las Isapres no es del todo legítimo pues la capacidad de pago, aun en el ámbito privado, no debe ser el único criterio para la adjudicación de un recurso básico, como lo es el cuidado sanitario. Por ello, al menos en lo que dice relación con el acceso a lo que se ha establecido como "mínimo sanitario" en Chile (GES-AUGE), los criterios que debieran primar son los de la universalidad y la no discriminación.

La última jurisprudencia ha dispuesto que cuando una persona no pueda acceder al derecho a la protección de la salud por problemas económicos, el Estado deberá actuar con preeminencia sobre los particulares en el entendido de que resulta fundamental garantizar que sea la necesidad, y no la capacidad de pago, la condición suficiente para ser beneficiario del derecho. Así, en el caso de Vivanco Garabito contra el Hospital Hernán 
Henríquez Aravena de Temuco, la Corte Suprema argumentó que "Si bien es cierto el tratamiento en cuestión se refiere a personas de 55 años y más, no por ello puede quedar sin recibir atención médica una persona por tener una edad inferior a ella" . Esto significa que la Constitución es un documento que garantiza positivamente el derecho a la salud, entregando al Estado la obligación de intervenir en su concreción real y oportuna.

Posteriormente, en el caso de Elena Vásquez Ibáñez contra FONASA, se establece que "el deber del Estado de Chile en materia de salud y el efectivo acatamiento de las garantías constitucionales de los derechos a la vida, integridad física y protección de la salud (...) obligan a proporcionar los medios para procurar el tantas veces citado medicamento (...) por lo que no le merece duda a esta Corte que debe proporcionársele el medicamento Herceptin $^{\circledR}$, en cuanto le va a permitir sobrellevar el grave cáncer que la afecta en mejores condiciones, aspirando a una sobrevida mayor"9. De este modo, se obliga al órgano público a ampliar, para el caso concreto, el contenido de la Guía Clínica respectiva, sobre la base de que la violación del principio de igualdad finalmente acarrearía también la violación del derecho a la vida.

El Tribunal Constitucional, a su turno, defiende que el derecho a la protección de la salud se halla sustancialmente ligado a otros atributos esenciales, como el derecho a la vida y a la integridad tanto física como psíquica, por lo que debe ser tutelado y promovido para infundir al ordenamiento legitimidad. "El Estado, por tanto, debe velar por la vida de las personas directamente, a través de su poder público, o 'indirectamente', por la vía de reconocer el derecho a la protección de la salud"10. En el mismo sentido ha declarado que los principales derechos sociales que la Constitución asegura a todas las personas son configurados a partir de la posibilidad de acceder a una determinada prestación y que la acción del Estado debe estar dirigida a garantizar el acceso de todos los habitantes al goce de dichas prestaciones ${ }^{11}$.

Lo anterior puede entenderse como una reacción directa a la Reforma Sanitaria del año 2005 que estableció un mínimo sanitario universalmente exigible a todas las instituciones -sean pública o privadas- con las garantías explícitas de acceso, calidad, oportunidad y cobertura financiera. Sin perjuicio de las dificultades que han existido para alcanzar el cumplimiento cabal de estas garantías ${ }^{12}$, el TC considera que la seguridad social moderna debe sustentarse en principios como el de igualdad, suficiencia, solidaridad y descentralización ${ }^{13}$.

\section{¿Qué experiencias comparadas pueden inspirar la reforma del modelo Isapres?}

¿Cómo facilitar a las Isapres el tránsito de un modelo de adjudicación actuarial a uno de tipo igualitario? Resulta interesante revisar legislación comparada como la "Tercera Directiva de Seguros no de Vida" (92/49/CEE) del Consejo de las Comunidades Europeas, que limita el alcance de la intervención gubernamental en los mercados privados de seguros de salud, con algunas excepciones. La directiva incluye una disposición especial (artículo 54) sobre los seguros de salud que actúan total o parcialmente como sustitutos de la cobertura de los sistemas nacionales de seguridad social. Conforme a esta norma, los estados miembros podrán adoptar disposiciones para proteger el "interés general" garantizando el acceso universal a los seguros con independencia de la edad o el estado de salud ${ }^{14}$. La lógica subyacente en el artículo 54.1 es la de permitir la regulación del seguro de salud privado que cumple una función de protección social al reemplazar al seguro público en la provisión del mínimo sanitario. Luego, se obliga a las aseguradoras privadas a permitir la inscripción abierta y la calificación comunitaria de los riesgos, con el fin de garantizar el acceso equitativo a la atención de la salud (se requerirá también un sistema de compensación de riesgos que permita reducir los incentivos para la selección y fomente la competencia basada en precio y calidad $)^{15}$.

Siguiendo esta normativa, la mayoría de los estados europeos han realizado reformas del sector sanitario considerando que la salud es un bien que genera beneficios a la sociedad toda y que el principio de solidaridad debiera expresarse por medio de subvenciones cruzadas, compensando unos (sanos, hombres, jóvenes y ricos) los mayores riesgos de otros (enfermos, mujeres, pobres y viejos $)^{16}$. En esta línea, el modelo sanitario del Reino Unido, que es un sistema pluralista, posee planes que aspiran a incrementar tanto la utilidad como la justicia en el sistema ${ }^{17}$. Se trata de un Servicio Nacional de Salud fundado en los principios de universalidad, exhaustividad y acceso gratuito que proporciona cuidado sanitario completo a 
todas las personas, independiente de su edad, clase social, sexo, posición geográfica etc. Y, por tanto, sólo de acuerdo con su necesidad sanitaria ${ }^{18}$.

A su turno, el modelo de Alemania posee un sistema legal de seguros sanitarios (llamados "fondos de enfermedad") para la mayoría de la población, permitiendo que el resto de las personas disponga de seguros privados ${ }^{19}$. Los fondos de enfermedad definen sus propias primas, aunque el Ministerio de Sanidad tiene la capacidad de regular el techo de ingresos por esta vía. El Libro V del Código Social define las prestaciones mínimas que deben proporcionarse y cómo debería regularse el sistema. Lo relevante, para el caso de Chile, es que tanto los fondos públicos como los seguros privados deben ofrecer pólizas que cubran el paquete de asistencia básica definido en la legislación, sin discriminación alguna por sexo o edad, tal y como lo dispone la Directiva 92/49 de la CCE.

Por último, puede destacarse el caso de los Países Bajos, que tienen un sistema sanitario legal basado en seguros que se financia a través de aportaciones a la seguridad social. Con arreglo a la ley, los seguros sanitarios son obligatorios y están subvencionados para las personas más pobres. En la actualidad, varios fondos de enfermedad sin fines de lucro se han transformado en aseguradoras privadas con fines de lucro que, a través de un "fondo de compensación de riesgos" que les garantiza no sufrir por la selección adversa de clientes, logran una sana competencia evitando la discriminación por sexo o edad (que está prohibida).

Como se puede apreciar de estos ejemplos, no hay duda que es posible cumplir con lo dispuesto por el Tribunal Constitucional y, a la vez, mantener vigente un sistema privado de salud eficiente y rentable. Pero para ello las modificaciones que se propongan a la ley 18.933 de Isapres debieran dar por superado un contenido normativo del tenor del antiguo artículo 38 ter, pues cualquier tipo de alza de precios en función de los factores prohibidos es inconstitucional y afecta los derechos a la igualdad, a la protección de la salud y a la seguridad social, reconocidos en la Constitución ${ }^{20}$.

\section{Conclusión}

Con el fin de modificar el actual modelo de distribución de cuidado sanitario, el sistema privado de salud requiere avanzar en la configu- ración de una reforma legislativa que logre dar cabal cumplimiento a las decisiones del Tribunal Constitucional instaurando un modelo universal e igualitario en, al menos, el espacio de aquello que se considera "mínimo sanitario", tal y como ocurre en la mayoría de los países de Europa.

De este modo, se debiera garantizar que las aseguradoras privadas ofrezcan un paquete básico de servicios en condiciones igualitarias, es decir, sin discriminación, aceptando a todos los candidatos con una prima única, y con independencia de su riesgo individual. Aunque es manifiesto que los mecanismos de financiación de ese sistema pueden discutirse, a la luz de los ejemplos comparados y las recomendaciones de la OMS, se puede proponer la incorporación de las calificaciones comunitarias de riesgos, los mecanismos de subvenciones cruzadas o el establecimiento de fondos solidarios de compensación de riesgo inter-isapres.

\section{Referencias}

1. Beauchamp T, Childress J. Principles of Biomedical Ethics. Sixth Edition. New York, Oxford University Press, 2009.

2. Daniels N. Is There a Right to Health Care and, if so, What Does it Encompass?, A Companion to Bioethics. UK, Blackwell Publishing, 2001.

3. Daniels N. Justice, Health, and Health Care, Medicine and Social Justice. Essays on the Distribution of Health Care, Oxford University Press, 2002: 17.

4. De Lora P, Zúñiga A. El derecho a la asistencia sanitaria. Un análisis desde las teorías de la justicia distributiva, Madrid, Iustel, 2009.

5. Zúñiga A. "Sistemas sanitarios y Reforma AUGE en Chile”. Revista Acta bioética 2007; 13 (2): 5.

6. Tribunal Constitucional. Rol 976, 26/6/2008 y Rol 1710, 6/8/2010.

7. Alexy R. Teoría de los derechos fundamentales, Madrid, Centro de Estudios Políticos y Constitucionales, 2002.

8. Corte Suprema. Rol: 1324-2010. 25/02/2010.

9. Corte Suprema. Rol: 9317-2009. 28/01/2010.

10. Tribunal Constitucional. Rol No $976 / 2008$.

11. Tribunal Constitucional. Rol No 1710/2010, 6/8/2010.

12. Zúñiga A. El derecho a la vida y el derecho a la protección de la salud en la Constitución: una relación necesaria. Revista del Centro de Estudios Constitucionales 2011; Año 9 (1).

13. Tribunal Constitucional. Rol No 1710/2010.

14. Mossialos E, McKee M. "EU Law and the Social Cha- 
racter of Health Care", Work \& Society, N 38, PIE-Peter Lang, 2002.

15. Mossialos E, Thomson S. Voluntary Health Insurance in the European Union. Directorate General for Employment and Social Affairs of the European Commission, London School of Economics and Political Science, London, 2002.

16. Saltman R. The Western European Experience with Health Care Reform. Copenhagen, WHO/EURO, 2002.

17. Vladeckadeck B, Fishman E. Unequal by Design: Health
Care, Distributive Justice, and the American Political Process. Medicine and Social Justice. Essays on the Distribution of Health Care. Oxford University Press, 2002.

18. Crisp R. Treatment According to Need: Justice and the British National Health Service. Medicine and Social Justice. Oxford University Press, 2002.

19. Lewis R, Álvarez-Rosete A, Mays N. How to Regulate Health Care in England. An International Perspective. Londres, King's Fund, 2006.

20. Tribunal Constitucional. Rol 97, 26/6/2008. 